\title{
STUDY OF THREE DIMENSIONAL LAMINAR FORCED CONVECTION OF NANO FLUID FLOW THROUGH ECCENTRIC RECTANGULAR ANNULAR DUCT
}

\author{
Amr G. Eltorky ${ }^{a *}$, Mohamed Elhelw ${ }^{b}$, Mohamed Fayed ${ }^{c}$, Abdelhamid Attia $^{d}$ \\ ${ }^{a}$ Faculty of Engineering, Mechanical Department, Pharos University In Alexandria, Egypt \\ ${ }^{b, c, d}$ Faculty of Engineering, Mechanical Department, Alexandria University, Egypt \\ *Corresponding author: amr.gaber@pua.edu.eg
}

\begin{abstract}
A b s t r a c t:Heat transfer through the horizontal eccentric annulus between eccentric rectangular ducts with different eccentricities has been calculated with various aspect ratio of inner duct. Boundary conditions are used with constant hot temperature on inner duct and constant cold temperature on the outer duct. Space between two ducts included $\mathrm{TiO}_{2}$-water Nano-fluid with different solid Volume fraction $(\varphi=0,2,5,10 \%)$. The eccentricity was changed with different values $(E=0.025,0.05,0.075 \mathrm{~m})$ in left direction and aspect ratio was changed with different value $(\mathrm{AR}=0.25,0.375,0.5)$. Results show that with an increase of aspect ratios, the average Nusselt number increases. Alsowith an increase in eccentricity value,the average Nusselt numberremains constant, and then an increase begins to occur. And the average Nusselt number increase due to an increase in nanoparticle concentration.
\end{abstract}

\section{Introduction}

Science and engineering include many applications such as a generator, solar heating system, ventilation ducts, atomic reactors, electrical motor, heating and cooling of electric cables. Convection heat transfer inside eccentric annular duct may be used in above applications. Lately, implementing the impact of eccentricity and aspect ratio on heat transfer has been an interesting topic and using Nanofluid to enhance the thermal conductivity of the fluid and other fluid properties. However, much exploring related to this subject has been managed by two dimensional circular annular duct or square annular duct and relatively little three dimensional rectangular annular duct studies have been carried out.Hussein Togun et. al [1] surveyed studies on natural, mixed and forced heat transfer to Nano-fluid and fluid flow in an annulus passage configurations, and the result show that Nusselt number values for the boundary condition of constant wall temperature are lower than those at uniform heat flux. Batra and Eissa [2] studied heat transfer by laminar and forced convection of a model fluid in an eccentric annulus. For same values of the cross-section parameters, a Sutterby model fluid has been found to yield a lower average Nusselt number as compared to that for a Newtonian fluid. Manglik and Fang [3] make a Numerical solutions for fully developed, laminar and heat transfer by forced convective in eccentric annulus are given with an insulated on an outer surface, result show Increasing the eccentricity and reducing the radius ratio of the annulus geometry results in large non-uniformities in the axial velocity and temperature fields.Matin [4] studied non-Newtonian power-law liquid (fluid) by natural convection of two dimensional in steady state assumption between two eccentric square horizontal ducts at a constant temperature. The results prove that with increasing $\mathrm{n}$ from 0.6 to 1.4 , observe that the mean Nusselt number decreases and decrease of heat transfer rate. Arefmanesh et. al [5] studied analysis of heat transfer between two horizontal square ducts and passage full of (water$\mathrm{TiO}_{2}$ ) Nano-fluid and observed from the results that, an increase in average Nusselt number due to an increase in volume fraction of particles. El-Maghlany et. al [6] studied natural convection through an open horizontal eccentric passage between two eccentric square ducts. Results mention that with an 
increase in eccentricity in upper and lower direction, a decrease occurs in convection heat transfer coefficient.Ciampi et. al [7] studied heat transfer by mixed convection flow through concentric and horizontal tube annular passage and observed that the Nusselt number at the bottom of the cylinder lower than at the top. Ingham and Patel [8] studied mixed convection in a vertical eccentric annular passage for the fully developed flow of a Bingham plastic. Khandekar and Moharana [9] using doubly and singly connected tube for different cross sections, applying two-dimensional technique, laminar flow, fully developed and estimating pressure drop through this passage. Macêdo et. al [10] studied Generalized Integral Transform Technique by using a compound of numerical-analytical solution and obtained for non-Newtonian fluids and laminar flow into doubly connected ducts.

Jeevan et. al [11] are focused on discussing the heat transfer attitude of a square spongy annulus subjected to outer cold temperature and inner hot temperature and it is noticed that the Nusselt number at the bottom is higher than at the top hot wall of the annular passage. Mahmoodi and Mazrouei [12] studied free convection flow and heat rate of composition $(\mathrm{Cu}-$ water) Nano-fluid through an annular passage between two square bodies and the results show that for every Rayleigh numbers without $\mathrm{Ra}=10^{4}$ the Nusselt number rises with an increase in the solid volume fraction of the particles.Gavara and Kanna [13] studied Laminar free convection between coaxial two vertical rectangular pipes and observed that The average Nusselt number of lower face is further than 2.4 times of Nusselt number averaged on other faces for the Grashof of this study.Xing et. al [14] studied laminar flow through the rectangular duct and how it is affected by the change in aspect ratio and result show that the Poiseuille number rise as the aspect ratio reduces. Shah and London [15] pointed out some works that dealt with laminar flow inside annular passages and various other types of doubly connected geometries.Moreover, as well governing equations for finite volume process are taken from book for Versteeg and Malalasekera [16] has documentary the book of the preamble to computational fluid dynamics. There are some rules for each program; ANSYS-FLUENT 16.0 is used in this research, which required guides are theory guides, user guides and tutorial [17]. Wibulswas [18] studied laminar flow heat transfer for rectangular ducts and equilateral triangular ducts of various aspect ratios with the thermal boundary conditions of constant wall temperature and constant heat input per unit length of the duct. Bartlett and Viskanta [19] studied the enhancement of forced convection in a heated duct full of with high thermal conductivity media.Heat transfer enhancement from a heated surface using porous structures has a potential application in electronics cooling.

\section{Nomenclature}

B Width of outer rectangle, $(\mathrm{m})$

b Width of inner rectangle, $(\mathrm{m})$

$\mathrm{Cp} \quad$ Specific heat at constant pressure, $(\mathrm{kJ} / \mathrm{kg} . \mathrm{K})$

$\mathrm{D}_{\mathrm{h}} \quad$ hydraulic diameter

g Acceleration of gravity, $\left(\mathrm{m} / \mathrm{s}^{2}\right)$

$\mathrm{H} \quad$ Height of outer rectangle, (m)

h Height of inner rectangle, $(\mathrm{m})$

$\mathrm{h}_{\text {avg }} \quad$ Average heat transfer coefficient, $\left(\mathrm{W} / \mathrm{m}^{2} \mathrm{~K}\right)$

L Length of duct, (m)

$\mathrm{Nu}_{\text {avg }} \quad$ Average Nusselt number,

P Pressure, $\left(\mathrm{N} / \mathrm{m}^{2}\right)$ 


$\begin{array}{ll}\mathrm{T} & \text { Temperature, }(\mathrm{K}) \\ \mathrm{t} & \text { Time, }(\mathrm{s}) \\ \mathrm{u}, \mathrm{v}, \mathrm{w} & \text { Velocity components in the } \mathrm{x}, \mathrm{y} \text { and } \mathrm{z} \\ \Delta \mathrm{T} & \text { Temperature difference, }(\mathrm{K}) \\ \mathrm{q}_{\mathrm{avg}} & \text { Average heat flux }(\mathrm{W} / \mathrm{m} 2)\end{array}$

\section{Subscripts}

$\begin{array}{ll}\text { avg } & \text { Average } \\ \mathrm{c} & \text { Cold } \\ \mathrm{f} & \text { Fluid } \\ \mathrm{h} & \text { Hot } \\ \mathrm{nf} & \text { Nano fluid } \\ \mathrm{p} & \text { Particle }\end{array}$
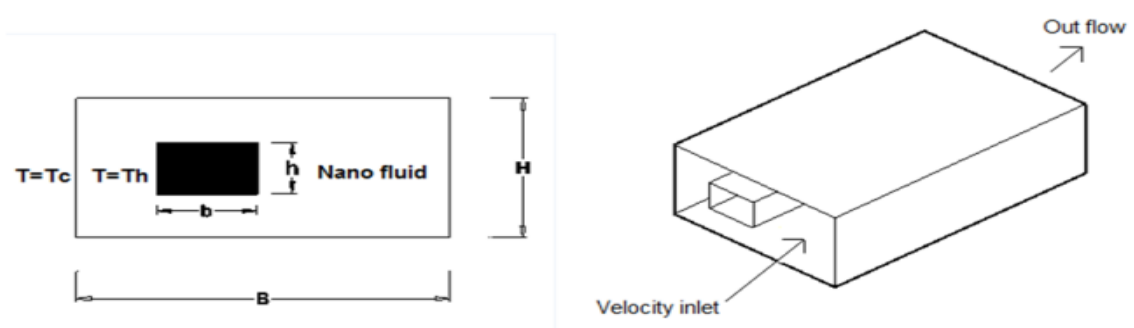

Fig. 1. Schematic of the eccentric rectangle with boundary conditions (current work).

\section{Scope and objectives}

Heat transfer calculations of forced convection in $\mathrm{TiO}_{2}$-water Nano-fluid from the surface of a heated horizontal inner duct to a parallel outer rectangular duct due to a few work in this area, are offered for different eccentricities, the solid volume fraction of particles and aspect ratio. The scope and objective of the current study are concentrated on finding the effects of eccentricities and aspect ratio on 3D annular rectangular ductassociated with forced convection through annulusas shown in Fig.1, resulting from the temperature difference between inner and outer duct.

\section{Problem formulation}

Consider the two eccentric ducts,which are shown in Fig.1.The height and the width of the outer duct are denoted by $\mathrm{H}$ and $\mathrm{B}$ respectively. Moreover, the height and the width of the inner duct are denoted by $h$ and $b$ respectively. The eccentricity $(\mathrm{E})$ is defined as a distance between the centers of duct, eccentricity ratio $\left(E_{R}=E / D_{h}\right)$. The aspect ratio of the annulus is known as $A R=h / H=b / B$. The length of the outer and the inner ducts orthogonal to the plane of the paper $=\mathrm{L}$. The outer and inner rectangular duct are keeping at different constant temperatures of $T_{c}$ and $T_{h}$, respectively, with $T_{h}>T_{c}$. The annular (the volume between the two rectangular duct) is filled with a composed of $\mathrm{TiO}_{2}$ spherical 
nanoparticles and water. The Nano-fluid is an incompressible flow, and the nanoparticles are supposed to be in thermal equilibrium together with the water. The eccentricity was changed with different values $(E=0.025,0.05,0.075 \mathrm{~m})$ in left direction and aspect ratio was changed with different value $(\mathrm{AR}=0.25,0.375,0.5)$. Moreover, there is no slip between the particles and water. Table 1 presented the physical properties of water and the nanoparticles. Thermal properties of Nano-fluid are a blend of $\mathrm{TiO}_{2}$ spherical nanoparticles and water can be calculated by equations included volume fraction of nanoparticles. The properties of the Nano-fluid $\left(\mathrm{TiO}_{2}\right.$ - water) are supposed to be constant.

Table.1. physical properties of the base fluid and nanoparticles [5]

\begin{tabular}{lcc}
\hline Physical propertieswaterNanoparticles $\left(\mathrm{TiO}_{2}\right)$ \\
\hline $\mathrm{C}_{\mathrm{p}}(\mathrm{J} / \mathrm{kg} \cdot \mathrm{K})$ & 4179 & 686.2 \\
$\rho\left(\mathrm{kg} / \mathrm{m}^{3}\right)$ & 997.1 & 4250 \\
$\mathrm{k}(\mathrm{W} / \mathrm{m} . \mathrm{K})$ & 0.613 & 8.9538 \\
$\beta \times 10^{5}\left(\mathrm{~K}^{-1}\right)$ & 21 & 0.9 \\
$\mu(\mathrm{kg} / \mathrm{m}-\mathrm{s}) 0.001003-$ &
\end{tabular}

\subsection{Thermophysical properties of the Nano-fluid}

The following equations proposed by A. Arefmanesh [5] included the density $\left(\rho_{\mathrm{nf}}\right)$, the thermal expansion coefficient $\left(\beta_{\mathrm{nf}}\right)$ and the specific heat capacity, $\left(\mathrm{Cp}_{\mathrm{nf}}\right)$ of the Nano-fluid respectively.

$$
\begin{gathered}
\rho_{n f}=(1-\phi) \rho_{f}+\phi \rho_{p} \\
\beta_{n f}=\frac{(1-\phi)(\rho \beta)_{f}+\phi(\rho \beta)_{p}}{(1-\phi) \rho_{f}+\phi \rho_{p}} \\
C_{p_{n f}}=\frac{(1-\phi) \rho_{f} C_{p_{f}}+\phi \rho_{p} C_{p_{p}}}{(1-\phi) \rho_{f}+\phi \rho_{p}}
\end{gathered}
$$

The Nano-fluid viscosity $\left(\mu_{\mathrm{nf}}\right)$ and thermal conductivity $\left(\mathrm{k}_{\mathrm{nf}}\right)$ of Nano-fluid are denoted by:

$$
\begin{aligned}
& \mu_{\mathrm{nf}}=\mu_{\mathrm{f}}\left(1+4.62 \phi+199.21 \phi^{2}\right) \\
& k_{n f}=k_{f}\left(1+4.82 \phi+125.62 \phi^{2}\right)
\end{aligned}
$$

The thermal diffusivity equation of the Nano-fluid $\alpha_{n f}$.

$$
\alpha_{\mathrm{nf}}=\frac{k_{n f}}{\left(\rho \mathrm{C}_{\mathrm{p}}\right)_{\mathrm{nf}}}
$$

The average Nusselt number on the outer surface based on hydraulic diameter of the annulus is evaluated from the following relation:

$$
\mathrm{Nu}_{\text {avg }}=\frac{q_{\text {avg }} D_{h}}{k_{n f} \Delta T}
$$

\subsection{Basic Governing Equations}

The components, $\mathrm{x}, \mathrm{y}$ and $\mathrm{z}$ of Continuity, momentum, and energy equations for the three-dimensional steady and laminar forced convection for Nano-fluid flow are given respectively.

The Three Dimensional continuity equation for fluid flow is given in Cartesian coordinates: 


$$
\frac{\partial \rho u}{\partial x}+\frac{\partial \rho v}{\partial y}+\frac{\partial \rho w}{\partial z}=0
$$

Three Dimensional Navier Stoke equations and energy equation for fluid flow are given in Cartesian coordinates:

$$
\begin{gathered}
-\frac{\partial \mathrm{P}}{\partial \mathrm{x}}+\mu\left[\frac{\partial^{2} \mathrm{u}}{\partial \mathrm{x}^{2}}+\frac{\partial^{2} \mathrm{u}}{\partial \mathrm{y}^{2}}+\frac{\partial^{2} \mathrm{u}}{\partial \mathrm{z}^{2}}\right]=\rho\left[\mathrm{u} \frac{\partial \mathrm{u}}{\partial \mathrm{x}}+\mathrm{v} \frac{\partial \mathrm{u}}{\partial \mathrm{y}}+\mathrm{w} \frac{\partial \mathrm{u}}{\partial \mathrm{z}}\right] \\
-\frac{\partial \mathrm{P}}{\partial \mathrm{y}}+\mu\left[\frac{\partial^{2} \mathrm{v}}{\partial \mathrm{x}^{2}}+\frac{\partial^{2} \mathrm{v}}{\partial \mathrm{y}^{2}}+\frac{\partial^{2} \mathrm{v}}{\partial \mathrm{z}^{2}}\right]=\rho\left[\mathrm{u} \frac{\partial \mathrm{v}}{\partial \mathrm{x}}+\mathrm{v} \frac{\partial \mathrm{v}}{\partial \mathrm{y}}+\mathrm{w} \frac{\partial \mathrm{v}}{\partial \mathrm{z}}\right] \\
\rho g_{\mathrm{z}}-\frac{\partial \mathrm{P}}{\partial \mathrm{z}}+\mu\left[\frac{\partial^{2} \mathrm{w}}{\partial \mathrm{x}^{2}}+\frac{\partial^{2} \mathrm{w}}{\partial \mathrm{y}^{2}}+\frac{\partial^{2} \mathrm{w}}{\partial \mathrm{z}^{2}}\right]=\rho\left[\mathrm{u} \frac{\partial \mathrm{w}}{\partial \mathrm{x}}+\mathrm{v} \frac{\partial \mathrm{w}}{\partial \mathrm{y}}+\mathrm{w} \frac{\partial \mathrm{w}}{\partial \mathrm{z}}\right] \\
\rho \mathrm{C}_{\mathrm{p}}\left[\mathrm{u} \frac{\partial \mathrm{T}}{\partial \mathrm{x}}+\mathrm{v} \frac{\partial \mathrm{T}}{\partial \mathrm{y}}+\mathrm{w} \frac{\partial \mathrm{T}}{\partial \mathrm{z}}\right]=\mathrm{k}\left[\frac{\partial^{2} \mathrm{~T}}{\partial \mathrm{x}^{2}}+\frac{\partial^{2} \mathrm{~T}}{\partial \mathrm{y}^{2}}+\frac{\partial^{2} \mathrm{~T}}{\partial \mathrm{z}^{2}}\right]
\end{gathered}
$$

Few assumptions are made such as, constant fluid properties, incompressible flow, radiation is neglected, laminar flow, and steady state conditions are assumed.

$$
\begin{gathered}
\mathrm{Gr}=\frac{\mathrm{g} \beta_{n f}(\Delta T) \mathrm{D}_{h}^{3}}{v_{n f}^{2}} \\
\operatorname{Re}=\frac{\rho_{n f} \mathrm{~V}_{\text {in }} \mathrm{D}_{h}}{\mu_{n f}} \quad \operatorname{Pr}=\frac{v_{\mathrm{nf}}}{\alpha_{n f}} \mathrm{Ri}=\frac{\mathrm{Gr}}{\mathrm{Re}^{2}}
\end{gathered}
$$

Where the Grashof Number (Gr), the Reynolds number (Re), the Prandtl number (Pr), inlet velocity $\left(\mathrm{V}_{\text {in }}\right)$ and Richardson number (Ri) to calculate if the flow is forced convection, natural convection or mixed convection. Richardson number has been lower than 1 to simulate forced convection dominant flow and in this case $\mathrm{Ri}=0.006$.

\subsection{Computer Program Validation}

To ensure that the FLUENT extractresults are in good agreement with previous work acomparison with a previous experimental result are made.
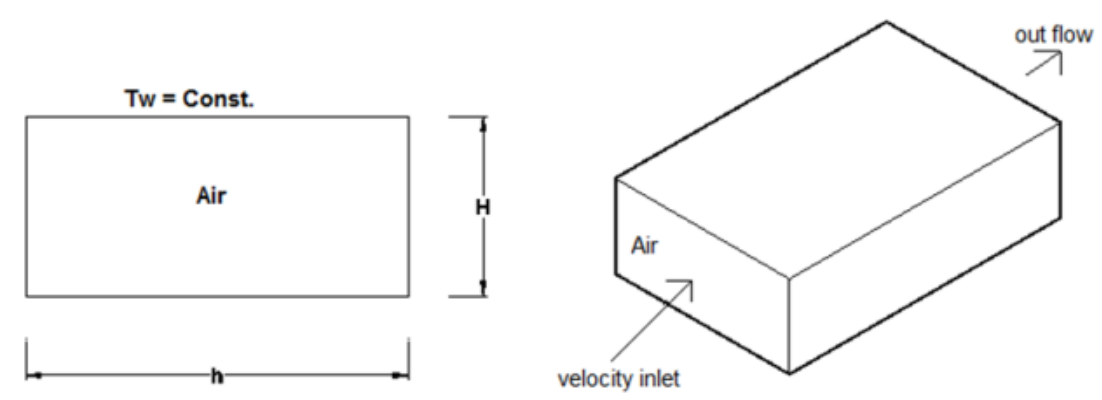

Fig.2.Schematic of rectangle duct by Wibulswas [18]

A comparison is used in the case of constant wall temperature in rectangle duct and the test section was made of copper which is a good heat conductor. Its dimensions were originally $2 \mathrm{ft}$. in length with aspect ratio $2,2 \times 1 \mathrm{in}^{2}$ in cross section and a wall of $1 / 8 \mathrm{in}$. in thickness were chosen and space filled by air as shown in Fig.2. From this comparison we observed that the various between the FLUENT result and experimental results [18] are between (0.5 to $7.5 \%)$ as shown in Fig.3. It can be 
noticed that the average Nusselt number of the present study is in good agreement with the Wibulswas [18]results.

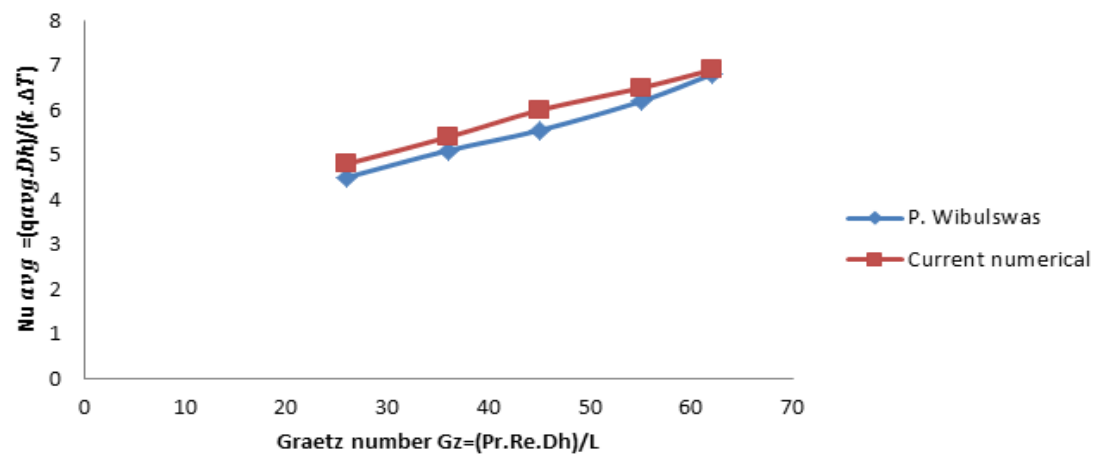

Fig.3. Average Nusselt number for laminar flow through a rectangle duct as a function of nondimensional Graetz number $(\mathrm{Gz})$ and validation of the current numerical with Wibulswas [18]

\subsection{Grid independence study}

In order to set a suitable grid for the present numerical solution, a grid independence study is managed for the forced convection heat transfer in the annulus between eccentric rectangular ducts shown in Fig.1. The aspect ratio is $\mathrm{AR}=1 / 2$, eccentricity ratio $\mathrm{E}_{\mathrm{R}}=0.375$ and the computation are completed for $\mathrm{TiO}_{2}$-water Nano-fluid with $\varphi=2 \%$ at $\Delta \mathrm{T}=65^{\circ} \mathrm{c}, \mathrm{Re}=10^{3}$ and $\mathrm{D}_{\mathrm{h}} / \mathrm{L}=0.011$. Five various grid system sizes, namely, $(9 \times 18,19 \times 38,29 \times 58,39 \times 78$ and $49 \times 98)$ are utilized for the numerical simulations. As shown in Fig.4 that the average Nusselt number on outer duct for these grids. It is observed from Fig. 4 that the appropriate results at less time a domain grid of $29 \times 58$ was adopted. Therefore, due to these results, a $29 \times 58$ grid system is utilized in all the subsequent numerical calculations.

\section{Grid independence}

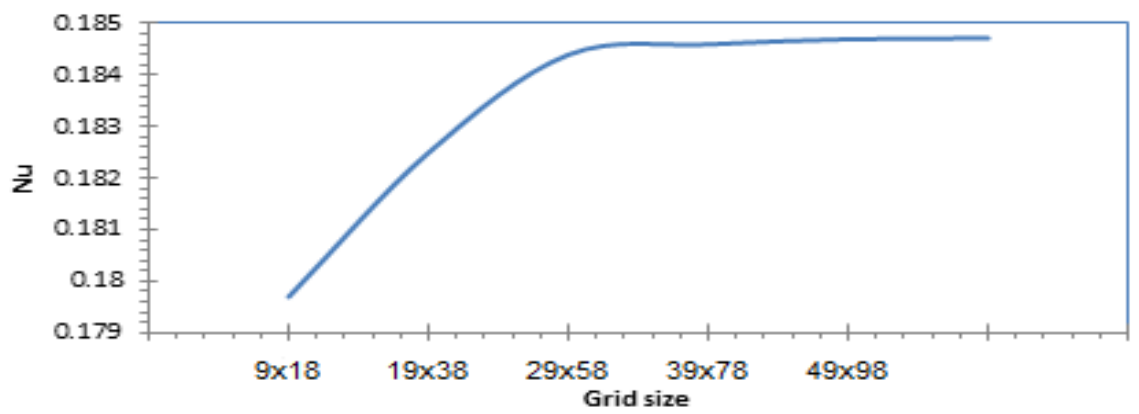

Fig.4.The average Nusselt number on the outer surface with different grid size

\section{Results and discussions}

Investigation of the effect of eccentricity and aspect ratio on heat transfer has become a subject of interest. The suggested code is used to study the laminar forced convection of Nano-fluid flow through the annular duct and heat transfer in the space between two eccentric rectangular ducts as shown in Fig.1, full of $\mathrm{TiO}_{2}$-water Nano-fluid. The results obtained are for a range of aspect ratios between 0.25 to 0.5 and a range of eccentricity from 0.025 to $0.075 \mathrm{~m}$, and solid volume fractions $\varphi=2$ 
$\%$ for aspect ratio effect case and eccentricity effect case, $\Delta \mathrm{T}=65^{\circ} \mathrm{c}, \mathrm{Re}=10^{3}, \mathrm{~L}=12 \mathrm{~m}, \mathrm{H}=0.2 \mathrm{~m}, \mathrm{~B}=0.4$ mwith several hydraulic diameter according to various aspect ratio. Fig. 5 to $7 \&$ Fig. 9 to 11 show the streamlines and the isotherms in the annulus with different aspect ratios and different eccentricities. The fluid is heated by the sides of the inner rectangular duct and extend as it shifts upward and the fluid is cooled by the sides of the outer rectangular duct as it shifts downward.

\subsection{Effect of aspect ratio (AR)}

The average Nusselt number was calculated on the outer surface of the duct at various aspect ratios of the duct from FLUENT numerical solution, Fig. 5, 6, 7 shows the fluid flow through the different aspect ratios at the outlet port, the aspect ratio varying from 0.25 to 0.5 and eccentricity $=0.05$ $\mathrm{m}$, in streamline figures two counter-rotating vortexes, a clockwise and a counterclockwise, are decided in the right and the left of the annulus, respectively. The heat convection taking space from the portion of the hot surface of the inner rectangle forms a streamline plume according to the flow of the fluid in the counter-rotating vortexes in this space as shown in Fig.5. By comparing streamlines at outlet cross section port of Fig. 5, 6, 7 with increasing aspect ratio with the same eccentricity, noted that the streamlines become more crowded adjacent to sides of the outer and the inner rectangle. Also, as Fig.7 shows, rotary vortex eyes will disappear in the left side of the space with increasing aspect ratio for the same eccentricity, rotary vortex eyes are converted from circular to ellipse cross section in the right side of the space with increasing aspect ratio. As far as the isotherms are involved, in some ways, isotherms distributed within the entire annulus with some special thermal boundary layers are created around the inner rectangle as shown in Fig.7. Furthermore, the thermal boundary layer thickness decrease and lead to an increasein heat transfer by increasing the aspect ratio. From Fig.8, it is observed that the convection heat transfer coefficient increasesby increasing the aspect ratio. This phenomenon occurs because with increasing the aspect ratio the gap between the ductsbecome small, then the fluid traps in the annulus left side and can't move freely. However, in annulus right side the fluid velocity increase and lead to an increase in average velocity and also average heat transfer coefficient.So,the average Nusselt number increases with increasing the aspect ratio due to an increase in temperatureprofile and also an increase in average temperature through the annulus. These observations mean that an increase in aspect ratio is associated with an increase in heat transfer rate.

A) Streamline

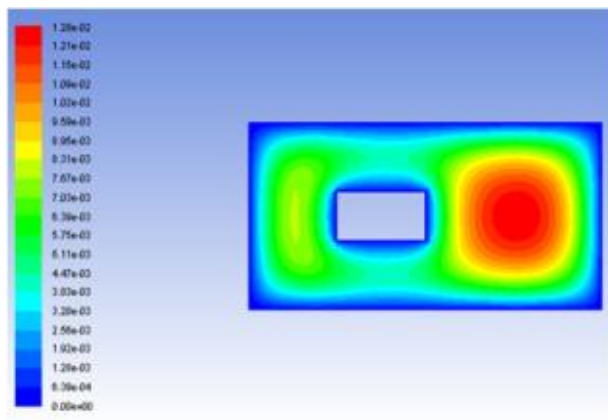

B) Isotherms

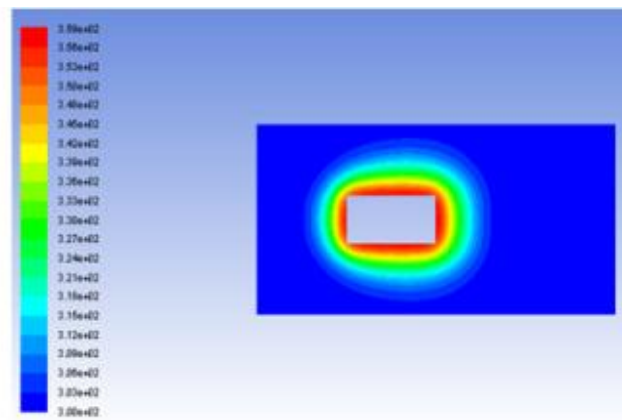

Fig.5. Streamline and Isotherms for $\mathrm{AR}=0.25 \& \mathrm{E}_{\mathrm{R}}=0.25$ 

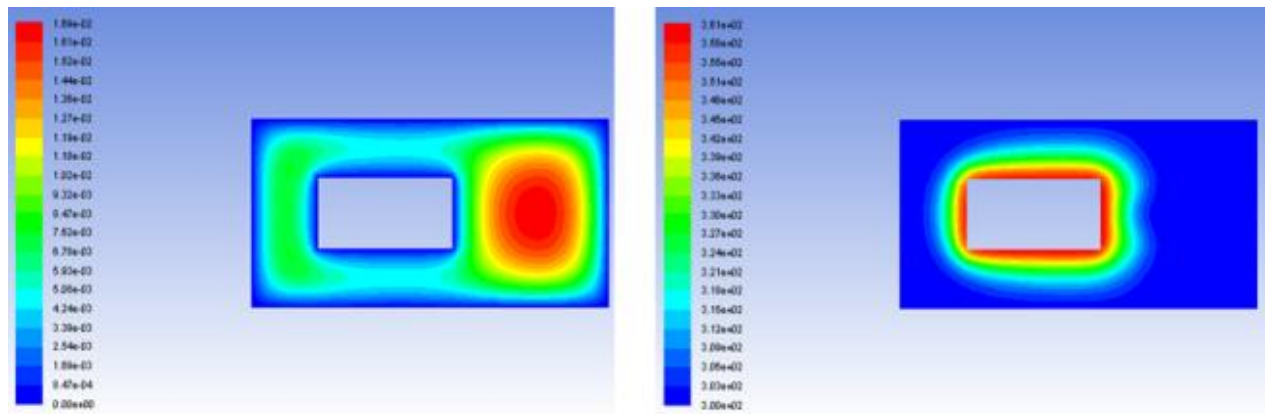

Fig.6.Streamline and Isotherms for $\mathrm{AR}=0.375 \& \mathrm{E}_{\mathrm{R}}=0.3$

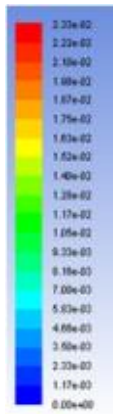
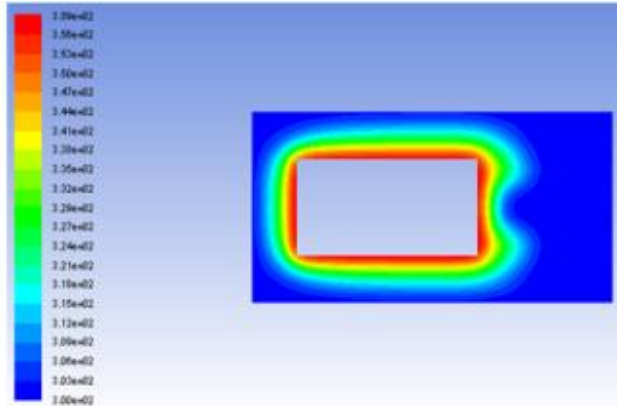

Fig.7.Streamline and Isotherms for $\mathrm{AR}=0.5 \& \mathrm{E}_{\mathrm{R}}=0.375$

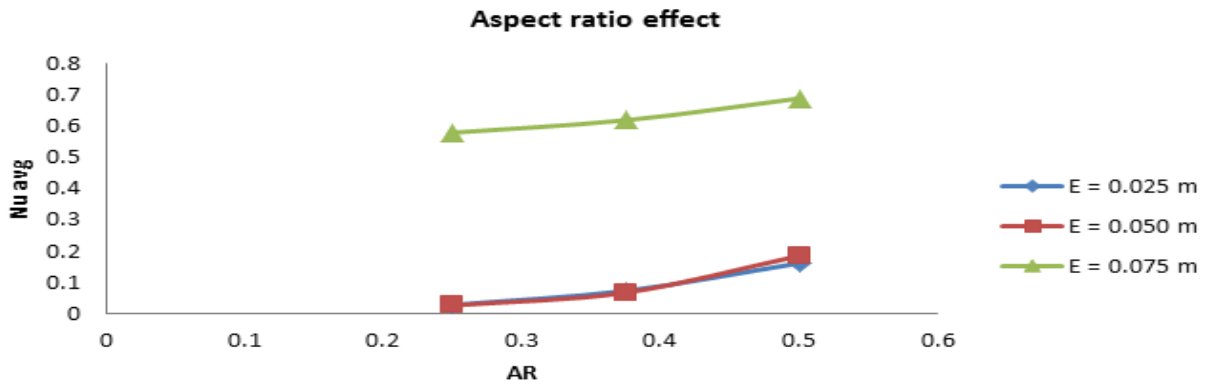

Fig.8.Effect of aspect ratio on the average nusselt number

\subsection{Effect of Eccentricity (E)}

Forced convection in an open-ended horizontal eccentric annulus between two eccentric horizontal rectangular ducts with different eccentricities has been studied with constant wall temperature. Fig. 9, 10, 11 show streamlines and isotherms at constant aspect ratio and different eccentricity at outlet cross section port of the annulus. The boundary conditions on inner and outer ducts are as follows: the constant hot temperature on the inner duct, the constant cold temperature on the outer duct. The eccentricity ratio was changed within a range between $\left(E_{R}=0.1\right.$ to 0.6$)$ in left direction for same aspect ratio. Fig.11 shows the flow it takes the right side path of the annulus and disappears from left side due to increasing eccentricity of the inner rectangle and lead to decreasing heat transfer on the left side but increasing on other sides. As far as the isotherms are involved, in some ways, isotherms distributed within the entire annulus and Fig. 11 shows how heat transfer increases on the sides by increasing eccentricity due to thermal boundary layer thickness. The results of the average Nusselt number on the outer rectangle are presented in Fig. 12 and indicate that with an increase in the 
value of eccentricity ratio, the convection heat transfer coefficient remains constant within a range of eccentricity ratio between zero and 0.35 . Eccentricity effect on heat transfer coefficientin this range not significantly different from those of concentric annuli, and then an increase begins to occur. However, the average convection heat transfer coefficient is very sensitive to eccentricity ratio for a value greater than 0.35 . The highest values of average Nusselt number are accomplished at the higher level of eccentricity. Because the narrow left section flow blockage results in increased velocities in the wider annular gap and also increase in heat transfer coefficient. As a result, the average Nusselt number remains constant before an increase begins to occur. These observations mean that an increase in eccentricity is associated with an increase in heat transfer rate.

\section{A) Streamline}

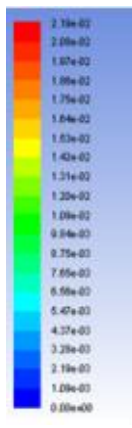

\section{B) Isotherms}

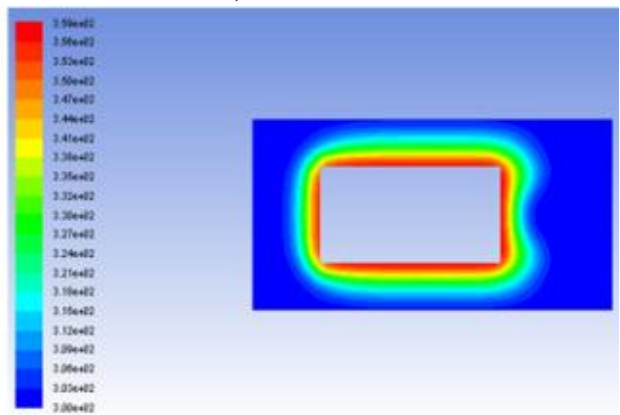

Fig.9.Streamline and Isotherms for $\mathrm{AR}=0.5 \& \mathrm{E}_{\mathrm{R}}=0.187$

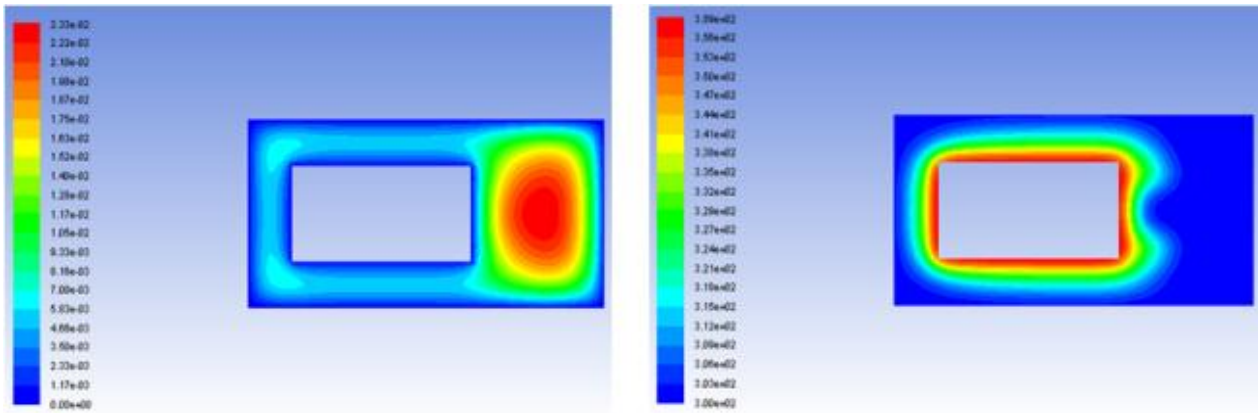

Fig.10.Streamline and Isotherms for $A R=0.5 \& E_{R}=0.375$
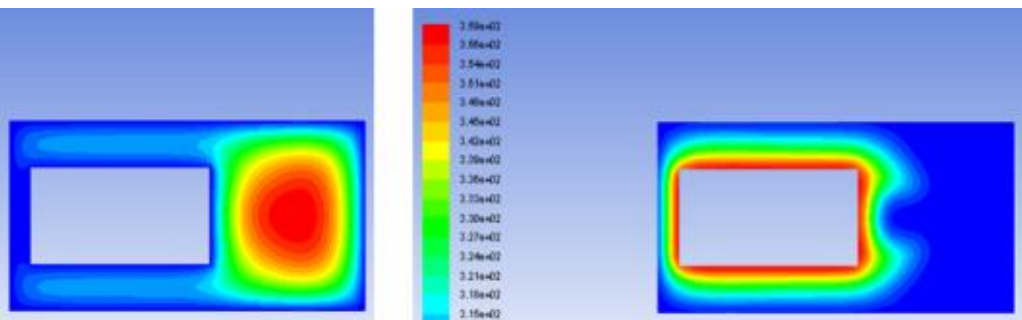

Fig.11. Streamline and Isotherms for $\mathrm{AR}=0.5 \& \mathrm{E}_{\mathrm{R}}=0.562$ 


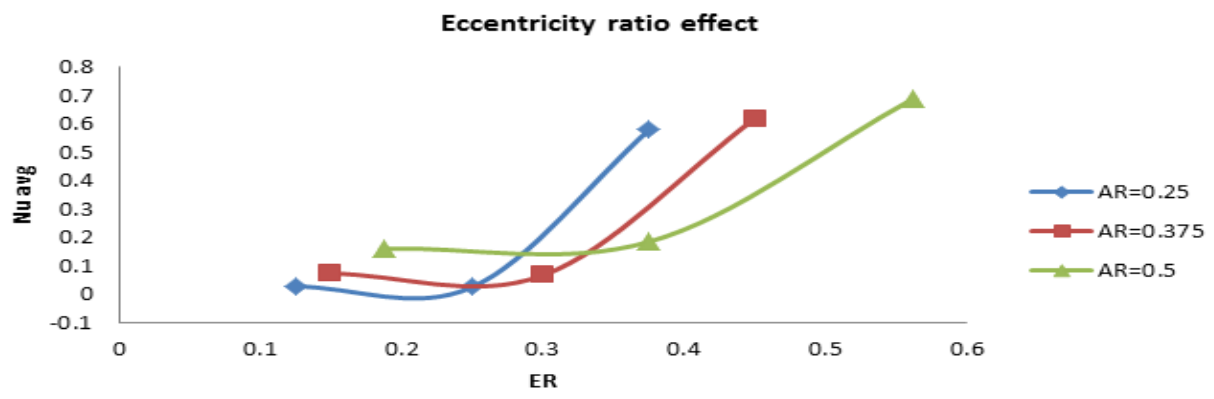

Fig.12.Effect of eccentricity on average nusselt number

\subsection{Effect of Particle Volume fraction $(\varphi)$}

Nano-fluids are comprised of Nano-sized solid particles that may change in size/shape in a liquid. The results of thermal conductivity presented show that an increase in temperature and volume concentrations is due to an increase in thermal conductivity of Nano-fluid. The aspect ratio is AR $=1 / 2$, eccentricity ratio is equal to 0.375 , and the computations are completed for $\mathrm{TiO}_{2}$-water Nano-fluid with solid Volume fraction $(\varphi)$ range between $0 \%$ and $10 \%$ and constant wall temperature on inner and outer duct at $\Delta \mathrm{T}=65^{\circ} \mathrm{c}$ and $\mathrm{D}_{\mathrm{h}} / \mathrm{L}=0.011$. The calculated data also showed that the viscosity of Nano-fluids increaseby increasing particle volume concentration.Finally, pure water had the lowest viscosity. The influence of nanoparticle concentrations on average Nusselt number is shown in Fig. 13. Results indicate, that the Nusselt number increases due to an increase in nanoparticle concentration given that the aspect ratio, eccentricity remain constant. The highest values of average Nusselt number are accomplished at higher level of concentration of solid nanoparticles which is related to an increase in thermal boundary layer profile.

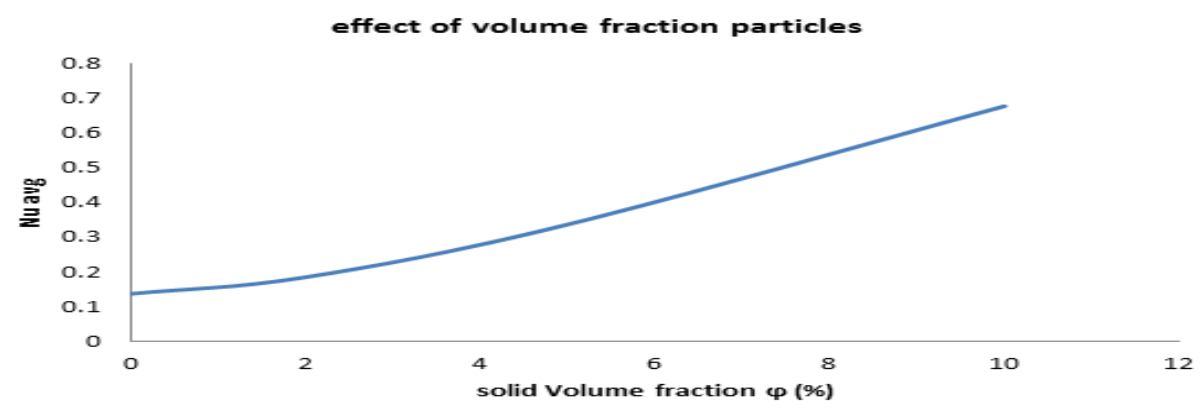

Fig.13. Effect of Solid Volume Fraction on Average Nusselt number

\section{Conclusions}

The heat transfer and fluid flow through two rectangular duct annuli filled with $\mathrm{TiO}_{2}$-water Nanofluid flow are presented to determine the effect of a number of variables. The finite volume method together with the SIMPLE algorithm is proceeded to solve the governing equations. The numerical procedure is verified via simulations, forced convection heat transfer in a differentially-heated rectangular cavity filled with $\mathrm{TiO}_{2}$-water.A variables study involving the effects of the annulus aspect ratio, eccentricity of the inner duct on horizontal axis to left side and heat transfer are conducted. The results show: 
1- The average Nusselt number on the outer duct is increase with increasing the aspect ratio. These observations mean that an increase in aspect ratio is associated with an increase in heat transfer rate.

2- Also, an increase in the value of eccentricity, the average Nusselt number remains constant and then an increase begins to occur. These observations mean that an increase in eccentricity is associated with an increase in heat transfer rate.

3- Nusselt number increase by increasing solid Volume fraction.

\section{References}

[1] Togun H, Abdulrazzaq T, Kazi SN, Badarudin A, Kadhum AAH, Sadeghinezhad E. A review of studies on forced, natural and mixed heat transfer to fluid and nano fluid flow in an annular passage 2014;39:835-56.

[2] Tobergte DR, Curtis S. Summary for Policymakers. In: Intergovernmental Panel on Climate Change, editor. Clim. Chang. 2013 - Phys. Sci. Basis, vol. 53, Cambridge: Cambridge University Press; 2013, p. 1-30.

[3] Manglik RM, Fang PP. Effect of eccentricity and thermal boundary conditions on laminar fully developed flow in annular ducts. Int J Heat Fluid Flow 1995;16:298-306. doi:10.1016/0142-727X(95)00030-T.

[4] Matin MH, Pop I, Khanchezar S. Journal of Non-Newtonian Fluid Mechanics Natural convection of power-law fluid between two-square eccentric duct annuli. J Nonnewton-Fluid Mech 2013; 197:11-23.

[5] Arefmanesh A, Amini M, Mahmoodi M, Najafi M. European Journal of Mechanics B / Fluids Buoyancy-driven heat transfer analysis in two-square duct annuli filled with a nanofluid.Eur JMech B/Fluids2012;33:95-104.

[6] El-maghlany WM, Sorour MM, Hozien O. Experimental study of natural convection in an annulus between two eccentric horizontal square ducts 2015;65:65-72.

[7] Ciampi M, Faggiani S, Grassi W, Tuoni G, Incropera FP. Mixed convection heat transfer in horizontal, concentric annuli for transitional flow conditions. Int J Heat Mass Transf 1987;30:833-41.

[8] Studies AM. M i x e d c o n ve c t i o n f l o w of a Bi plastic in an e c c e n t r i c annulus 1994;15:132-41.

[9] Appl M, Engrg M. Generalized formulation for estimating pressure drop in fully-developed laminar flow in singly and doubly connected channels of non-circular. Comput Methods Appl Mech Eng 2013;259:64-76.

[10] Monteiro ER, Macêdo EN, Quaresma JNN, Cotta RM. International Journal of Heat and Mass Transfer Laminar flow and convective heat transfer of non-Newtonian fluids in doubly connected ducts. Int J Heat Mass Transf 2010;53:2434-48.

[11] Anjum I, Al-rashed AAAA, Ahmed NJS, Kamangar S, Jeevan K. International Journal of Heat and Mass Transfer Natural convection in a square porous annulus. Int J Heat Mass Transf 2012;55:7175-87.

[12] Mahmoodi M, Mazrouei S. Superlattices and Microstructures Natural convection in a square cavity containing a nanofluid and an adiabatic square block at the center. Superlattices Microstruct 2012;52:261-75.

[13] Gavara M, Kanna PR. Study of conjugate natural convection between vertical coaxial rectangular cylinders . Int Commun Heat Mass Transf 2012;39:904-12.

[14] Xing D, Yan C, Wang C, Sun L. Progress in Nuclear Energy A theoretical analysis about the effect of aspect ratio on singlephase laminar fl ow in rectangular ducts. Prog Nucl Energy 2013;65:1-7.

[15] Irvine TF, Hartnett JP. Edited by FORCED CONVECTION. n.d. shah and london.

[16] H. K. Versteeg and W. Malalasekera, "Introduction to computational fluid dynamics - Finite Volume Method" 1995.

[17] ANSYS, Inc. , "Fluent Theory Guide”.

[18] Wibulswas, P. Laminar-flow heat-transfer in non-circular ducts (Doctoral dissertation, University of London).

[19] R. F. Bartlett, Raymond Viskanta. Enhancement of Forced Convection in an Asymmetrically Heated Duct Filled with High Thermal Conductivity Porous Media 